\title{
An in silico platform for the design of heterologous pathways in nonnative metabolite production
}

\author{
Sunisa Chatsurachai ${ }^{1}$, Chikara Furusawa ${ }^{2,3^{*}}$ and Hiroshi Shimizu ${ }^{3^{*}}$
}

\begin{abstract}
Background: Microorganisms are used as cell factories to produce valuable compounds in pharmaceuticals, biofuels, and other industrial processes. Incorporating heterologous metabolic pathways into well-characterized hosts is a major strategy for obtaining these target metabolites and improving productivity. However, selecting appropriate heterologous metabolic pathways for a host microorganism remains difficult owing to the complexity of metabolic networks. Hence, metabolic network design could benefit greatly from the availability of an in silico platform for heterologous pathway searching.
\end{abstract}

Results: We developed an algorithm for finding feasible heterologous pathways by which nonnative target metabolites are produced by host microorganisms, using Escherichia coli, Corynebacterium glutamicum, and Saccharomyces cerevisiae as templates. Using this algorithm, we screened heterologous pathways for the production of all possible nonnative target metabolites contained within databases. We then assessed the feasibility of the target productions using flux balance analysis, by which we could identify target metabolites associated with maximum cellular growth rate.

Conclusions: This in silico platform, designed for targeted searching of heterologous metabolic reactions, provides essential information for cell factory improvement.

\section{Background}

Recognizing the potential depletion of petroleum resources, researchers have become increasingly interested in production of fuels and industrial chemicals by microorganisms [1-3]. Such biosnythesized materials include fuels, plastics, polymers, food additives, feed additives, solvents and drugs [4-6]. For example, ethanol and higher alcohols are used as fuels and solvents in a wide variety of chemical processes [7]. 1,3-propanediol forms the basis of polymers such as polytrimethylene terephthalate (PTT) [8], while isoprene is an intermediate metabolite in the production of cis-1,4-polyisoprene, a synthetic of natural rubber [9]. To produce such industrially useful materials, modifications of host metabolic systems are generally required. Target metabolites are

\footnotetext{
* Correspondence: chikara.furusawa@riken.jp; shimizu@ist.osaka-u.ac.jp ${ }^{2}$ Quantitative Biology Center, RIKEN, 6-2-3 Furuedai, Suita, Osaka 565-0874, Japan

${ }^{3}$ Department of Bioinformatics Engineering, Graduate School of Information Science and Technology, Osaka University, 1-5 Yamadaoka, Suita, Osaka 565-0871, Japan

Full list of author information is available at the end of the article
}

frequently produced by incorporating heterologous metabolic pathways into well-characterized host microorganisms, such as Escherichia coli, Saccharomyces cerevisiae, and Corynebacterium glutamicum [10-15]. However, the selection of suitable heterologous metabolic pathways for host organisms is hindered by metabolic network complexity. Although copious data on metabolic reactions have been amassed in the literature and in public databases, such as KEGG [16], BRENDA [17], and ENZYME [18], constructing a target production pathway from a host metabolic network while maintaining the required metabolic balances in the host (e.g., nicotinamide adenine dinucleotide (NADH) production/ consumption) requires a researcher's experience and intuition. Thus, the development of an appropriate in silico platform will enhance industry-focused metabolic network design by providing possible heterologous pathways for target metabolite production.

In recent years, several in silico heterologous pathway search methods have been proposed and used in target metabolite production [19-30]. Some of these predict

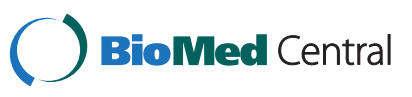


metabolic pathways based on chemical transformation patterns between the substrate and the product $[19,20,24,25]$. For example, PathMiner [19] heuristically determines metabolic pathways from known enzymecatalyzed transformations, by minimizing pathway costs. PathPred [29] extracts biochemical structural transformation patterns from databases, from which plausible pathways can be constructed even if no reactions that directly generate the target metabolites are known. By supplying information about reactions, PathPred enables the user to create a metabolite that is structurally similar to the target.

Several graph-based methods for heterologous pathway search are also available [21-23,26,28,30]. OptStrain [30] utilizes mixed integer linear programming to identify heterologous reactions, producing a target that satisfies the stoichiometric balance while minimizing the number of heterologous reactions. Following stoichiometric addition of the heterologous reactions, the OptKnock [31] algorithm maximizes the target productivity. As another example, novel metabolic routes have been efficiently screened by probabilistic selection of metabolic pathways [27]. Although several methods exist for screening heterologous pathways of target metabolite production, there remains a lack of consensus on how to choose heterologous pathways and host microorganisms for target production. Heterologous reaction screening generally requires extensive calculations; thus, it is difficult to compare the screening results. In this study, to avoid such calculations, we developed a simple in silico screening platform to identify feasible heterologous pathways of nonnative target metabolite production.

We first developed a pathway search algorithm that identifies the shortest pathway between a host metabolic network and target metabolites as heterologous reactions are added. Using this algorithm, we screened all producible target metabolites listed in databases by adding heterologous reactions to host microorganisms. For all producible target metabolites, we then estimated the production yields using flux balance analysis (FBA), assuming steady-state conditions and maximum biomass production rate. By analyzing the entire list of producible target metabolites in several different hosts, we selected a set of rational heterologous pathways and host microorganisms that will likely produce desired targets.

\section{Methods}

\section{Construction of an in-house database of metabolic reactions}

All known metabolic reactions were considered as candidate heterologous reactions that could be added to the host metabolic network. We first constructed an inhouse database of metabolic reactions from data stored in the KEGG ligand section [16] and BRENDA [17] databases. All metabolic reaction information regarding genes, enzymes, pathways, and organisms in the KEGG database was collected into the database, which was developed using PostgreSQL 9.0 (The PostgreSQL Global Development Group). The Michaelis-Menten constants $\left(K_{m}\right)$ of the enzymatic reaction data were retrieved from BRENDA [17]. We also used Python scripts to access the in-house database.

\section{Genome-scale metabolic model of host microorganisms}

In this study, we adopted 3 host microorganisms widely used in industry; namely, E. coli, C. glutamicum, and S. cerevisiae. E. coli has been exploited for such industrially valuable compounds as $\mathrm{L}$-phenylalanine, L-tyrosine, 1butanol and 1,2-propanediol [32-34]. C. glutamicum is widely used in amino acid production [35]. S. cerevisiae is an important producer of alcohols and organic acids such as lactate [36]. These organisms are ideal hosts of bioengineered products since they exhibit high growth activity under various conditions and are easily genetically manipulated $[37,38]$.

We used genome-scale metabolic models of $S$. cerevisiae (iMM904) [39], E. coli (iJR904) [40], and C. glutamicum [41], based on earlier metabolic constructions with slight modifications. Because our pathway search algorithm uses the heterologous reactions listed in the KEGG database, all metabolite IDs in the earlier genome-scale metabolic models were converted to the KEGG compound ID format using metabolite name matching by manual checking.

\section{Heterologous pathway identification for target production}

We developed an algorithm to identify heterologous reaction(s) producing a target metabolite within a host microorganism. The algorithm expands the host metabolic network by sequentially adding heterologous metabolic reactions from our in-house database. The heterologous pathway identification procedure is as follows:

1. A set of metabolites $M_{0}$ and a set of metabolic reactions $R_{0}$ are defined as those present in the genome-scale metabolic network of the host microorganism.

2. From the in-house database, heterologous reactions that satisfy the following conditions are collected: (i) the reaction does not exist in $R_{0}$, and (ii) it can produce metabolites that do not exist in $M_{0}$ from a metabolite in $M_{0}$. A set of these heterologous reactions is defined as $R_{1}$, and a set of metabolites produced by reactions in $R_{1}$ is defined as $M_{1}$.

3. In the same way, $R_{\mathrm{i}}$ is the set of reactions not present in $\left\{R_{0}, R_{1}, \ldots, R_{i-1}\right\}$ which can produce 
metabolites not existing in $\left\{M_{0}, M_{1}, \ldots, M_{i-1}\right\}$ from metabolites included in those sets. This expansion procedure is iterated until no further reaction is connectable to the expanded metabolic network.

If a target metabolite is included in a nonnative metabolite set $M_{i}$, we can identify a set of heterologous reactions that are necessary to produce the target metabolite. For simplicity, all metabolic reactions in the database were assumed to be reversible. Of course some reactions are known to be irreversible, such as the carboxylation and decarboxylation reactions classified by Nomenclature Committee of the international Union of Biochemistry and Molecular Biology (NC-IUBMB) [42]. However, for the majority of reactions in the database, directional information is limited and thus the reversibility of the reactions is difficult to judge. By assuming that all reactions are reversible, we avoid the risk of missing important heterologous pathways due to misjudgment of their reaction reversibility. Our strategy here is to initially screen all possible heterologous pathways regardless of reaction irreversibility, then decide whether the predicted pathway is plausible based on physiological knowledge of the reaction irreversibility.

\section{Flux balance analysis}

FBA is based on a genome-scale metabolic model and optimization of a specific objective flux by linear programming $[43,44]$. We used FBA to estimate the metabolic flux profile of metabolic networks expanded with heterologous reactions. A pseudo-steady state is assumed, that is, the net sum of all production and consumption fluxes for each internal metabolite is zero. In matrix notation, this condition is represented as $S \cdot v=0$, where $S$ is the stoichiometric matrix representing the stoichiometry of metabolic reactions in the network and $v$ is the vector of metabolic fluxes. In FBA, the flux profile (constrained by steady state) is determined by optimizing a specific objective function. The biomass production flux is one of several widely used objective functions that can be maximized. The flux profiles obtained by maximizing biomass production fluxes are known to be well correlated with those obtained experimentally [39-41,45].

In this study, the coefficients of metabolites representing biomass production flux were extracted from earlier studies [39-41]. We employed another objective function, the production flux of the target metabolite, to judge whether the target metabolite was producible by the metabolic network. In all of the FBA simulations in this paper, glucose was chosen as the sole carbon source and the following external metabolites were allowed to freely transport through the cell membrane: $\mathrm{CO}_{2}, \mathrm{H}_{2} \mathrm{O}$,
$\mathrm{SO}_{4}$ or $\mathrm{SO}_{3}$, and $\mathrm{NH}_{3}$. All calculations were performed using MATLAB 2009b (MathWorks Inc., Natick, MA). The linear programming problem was solved using GLPK 4.34 (GNU Linear Programming Kit) [46] via the MATLAB interface.

\section{Results and discussion}

\section{Identification of heterologous pathway(s)}

7,769 metabolic reactions and 6,635 metabolites (shown in the Additional file 1) from 1,139 species were collected from the KEGG database and deposited in our inhouse database. To screen for target metabolites that could be produced by our host microorganisms $S$. cerevisiae, E. coli, and C. glutamicum, we iteratively expanded the host metabolic network by adding heterologous metabolic reactions as described in the Methods section. Figure 1 displays the number of nonnative metabolites connected to the host metabolic network as a function of the number of heterologous reactions. Fewer than 33 heterologous reactions are required to connect 3,154, 3,244 , and 3,112 nonnative metabolites to the host metabolic networks of S. cerevisiae, E. coli, and C. glutamicum respectively.

The list of metabolites connected to the host metabolic networks is presented in the Additional files 2, 3, 4. To this list, we added the $K_{\mathrm{m}}$ values of heterologous enzymes. Knowing the $K_{\mathrm{m}}$ assists in deciding which heterologous enzymes originating from various organisms should be introduced to the host. The names of organisms in the BRENDA database displaying minimum $K_{\mathrm{m}}$ of the corresponding heterologous enzymes are also listed [17], since the enzyme from this organism is expected to have highest affinity among the orthologous enzymes to the corresponding substrate. Importantly, these identified heterologous reactions of nonnative metabolite production agreed well with those widely used in metabolic engineering and which are important to the industry (Table 1 ), such as isoprene, $\alpha$-farnesene, poly- $\beta$ hydroxybutyrate (PHB), and cadaverine.

As an example, the production pathways of 1,3-propanediol (C02457) by E. coli and S. cerevisiae, which were adopted in earlier studies [52,53], are shown in Figure 2. In the previous studies, $\mathrm{C} 02457$ production proceeded via conversion of glycerol to 3-hydroxypropanal using glycerol dehydratase (encoded by dhaB1-B3). 1,3-Propanediol was then produced, aided by 1,3-propanediol oxidoreductase (encoded by dhaT). In this study, the screened heterologous pathways for $\mathrm{C} 02457$ production exactly matched those of the earlier studies. In E. coli, the screened production pathways of isoprene, $\alpha$-farnesene, and PHB derived by our algorithm were also identical to those of the earlier studies, while similar heterologous genes introduced to the alternative hosts (C. glutamicum and $S$. cerevisiae) additionally produced these targets 


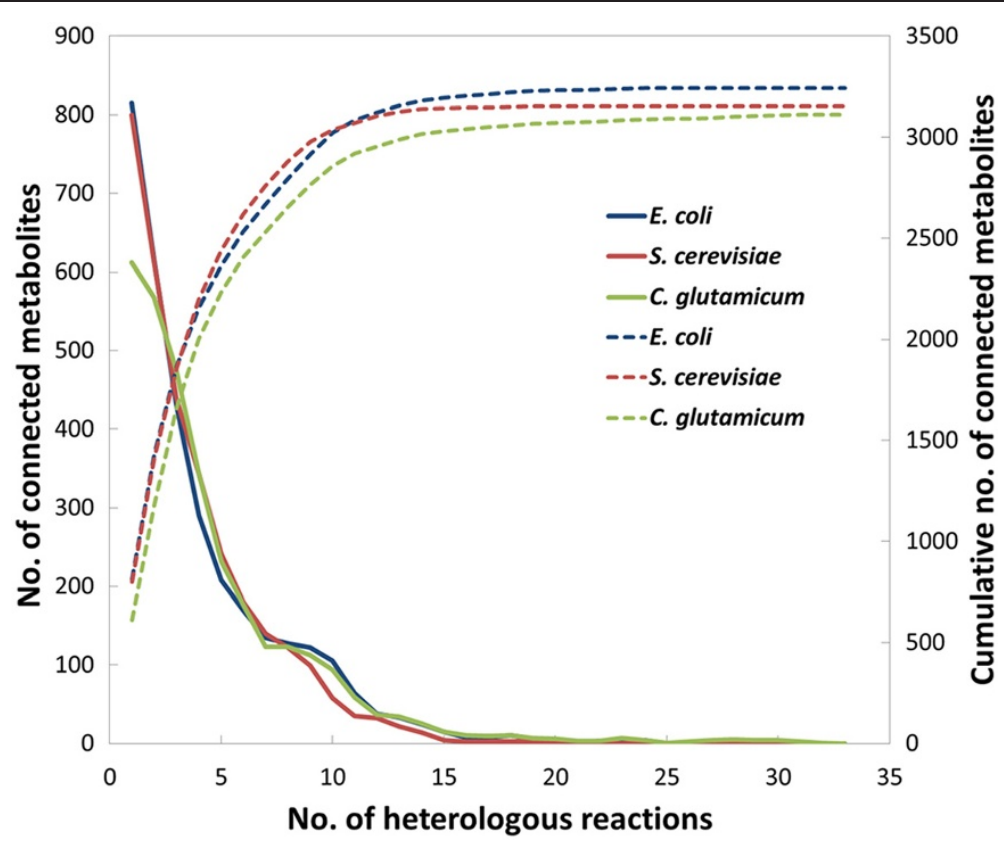

Figure 1 Number of connected nonnative metabolites produced by heterologous reactions in $\mathbf{3}$ host microorganisms. The first vertical axis (solid line) shows the number of connected metabolites in each iteration, while the second vertical axis (dotted line) shows the cumulative number of the connected metabolites.

(see Table 1). Moreover, both reported and alternative production pathways were screened by our algorithm. For instance, we found that E. coli cells can produce (R)-propane-1,2-diol when methylglyoxal reductase and lactaldehyde reductase are added to the metabolic network, which has not been reported to date. Similar alternative pathways were found for the production of itaconate, cis, cis-muconate, and 2,3-dihydroxybenzoate. These results suggest that our algorithm successfully identified the metabolic reactions necessary for the target productions and could assist in screening for potential host cells.

Next, we used glucose as a carbon source to investigate whether these nonnative metabolites are producible by FBA simulations. In this simulation, the production flux of each nonnative metabolite was treated as an objective function to be maximized under the steady-state assumption. When the maximum production flux of a nonnative metabolite is zero, this metabolite is nonproducible under the given condition.

We calculated the maximum production fluxes of all connectable nonnative metabolites. $28 \%$ of the connectable nonnative metabolites of E. coli could not be produced using glucose as a sole carbon source. Similarly, $33 \%$ of the connectable nonnative metabolites of S. cerevisiae and $16 \%$ of the connectable nonnative metabolites of C. glutamicum were non-producible under this condition. These non-producible metabolites were identified by their tendency to disconnect when glycolysis formed the central metabolic pathway. In E. coli, these metabolites included trans-aconitate (C02341), butyrate (C00246), acetoacetate (C00164), and L-lactaldehyde (C00424).

\section{Evaluation of production feasibility}

To evaluate the feasibility of nonnative target metabolite production, we performed FBA simulations under conditions of maximizing biomass production following heterologous reaction expansion of the genome-scale metabolic model. Metabolic flux profiles calculated at maximum biomass production rates have been shown to closely represent those in real microorganisms [45,59-62]. Such agreement may be explained by the growth optimization of microorganisms through evolutionary dynamics [63]. Furthermore, for the mutant strains constructed in the laboratory, the cells could achieve the near-optimal metabolic state calculated by the FBA simulation after long-term cultivation [64-67], via the selection of faster growing cells. Thus, we can expect that if a nonnative target metabolite is produced in the FBA simulation under maximized biomass production, that target may be feasibly manufactured.

In Figure 3, we plot the number of target metabolites produced under maximized biomass production, versus the number of heterologous reactions necessary for metabolite production. We set a threshold yield (1\%) to identify the produced metabolites because the production yields of some metabolites were positive but extremely small. Sometimes the FBA solution was undetermined under biomass maximization conditions; 
Table 1 Examples of nonnative metabolites for which our algorithm detected heterologous reactions matching those of previous studies

\begin{tabular}{lll}
\hline Compound & KEGG Heterologous reaction(s) from & ReferenceEvaluation of in silico design \\
(synonym separated by a & ID the literature
\end{tabular}

(synonym separ

ID the literature

\section{Isoprene; \\ 2-methyl-1,3-butadiene}

a-Farnesene

C16521 Introduced ispS gene from

Populus nigra to Escherichia coli

C09665 Introduced farnesene synthase from plant to $E$. coli

Poly- $\beta$-hydroxybutyrate; PHB C06143 Introduced phbC and phbB from Streptomyces aureofaciens to E. coli

Cadaverine;

1,5-pentanediamine

1,5-diaminopentane

Amorpha-4,11-diene

Propane-1,3-diol;

1,3propanediol; trimethylene

glycol

\section{Ethanol;}

ethyl

alcohol; methylcarbinol

(R,R)-Butane-2,3-dio

(R,R)-2,3-Butanediol;

(R,R)-2,3-Butylene glycol

(R)-Propane-1,2-dio

(R)-1,2-propanediol,

(R)-propylene glycol

\section{Itaconate;}

itaconic acid

methylenesuccinic acid

cis,cis-Muconate;

cis,cis-hexadienedioate;

cis,cis-2,4-hexadienedioic

acid

Adipate;

adipic acid; hexanedioate;

hexan-1,6-dicarboxylate pneumonia to E. coli.
C01672 Introduced IdcC from E. coli to C. glutamicum

C16028 Introduced AMS1 from the plant Artemisia annua L. to $E$. coli

C02457 Introduced glycerol dehydratase and 1,3-propanediol

oxidoreductase from Klebsiella

C00469 Introduced pyruvate decarboxylase and alcoho dehydrogenase genes from Zymomonas mobilis to C. glutamicum

C03044 Introduced acetolactate decarboxylase and butanediol dehydrogenase genes to $E$. col

C02912 Introduced glycerol dehydrogenase gene from Klebsiella pneumonia and used aldehyde dehydrogenase to

produce product in $E$. coll

Introduced glycerol dehydrogenase and methylglyoxal synthase genes from $E$. coli to $S$. cerevisiae

C00490 No information

C02480 Introduced aroZ, aroY, and catA to E. coli [58]

C06104 Introduced aroZ, aroY, and catA to $E$. coli for producing cis, cismuconate and then convert to adipic acid by chemical synthesis
[47] Identical reaction found in E. coli and in Saccharomyces cerevisiae and Cerevisiae glutamicum as the host

[13] Identical reaction found in E. coli and in S. cerevisiae and C. glutamicum as the host

[48] Identical reaction found in E. coli and in S. cerevisiae and C. glutamicum as the host

[35,49] Identical reaction found in C. glutamicum and in S. cerevisiae as the host

[50,51] Identical reaction found in E. coli and S. cerevisiae and in C. glutamicum as the host

[52,53] Identical reaction found in E. coli and in S. cerevisiae as the host

[54] Identical reaction found in C. glutamicum as the hos

[55] Identical reaction found in E. coli as the host

[56] Alternative pathway found to produce target by adding methylglyoxal reductase and lactaldehyde reductase to $E$. coli

[57] Alternative pathway found to produce target by adding methylglyoxal reductase and lactaldehyde reductase to $S$. cerevisiae

EC 4.2.1.4-citrate dehydratase and EC 4.1.1.6-aconitate decarboxylase were found to be added to E. coli as the host

Alternative pathways from antharnilate or 2,3-dihydroxybenzoate to produce catechol, which is a substrate for cis,cis-muconate production

[58] Alternative pathway found to produce the target by adding 5 heterologous reactions to $E$. coli or $C$. glutamicum as the hosts (see Additional files 5 and 6 for enzyme information) 

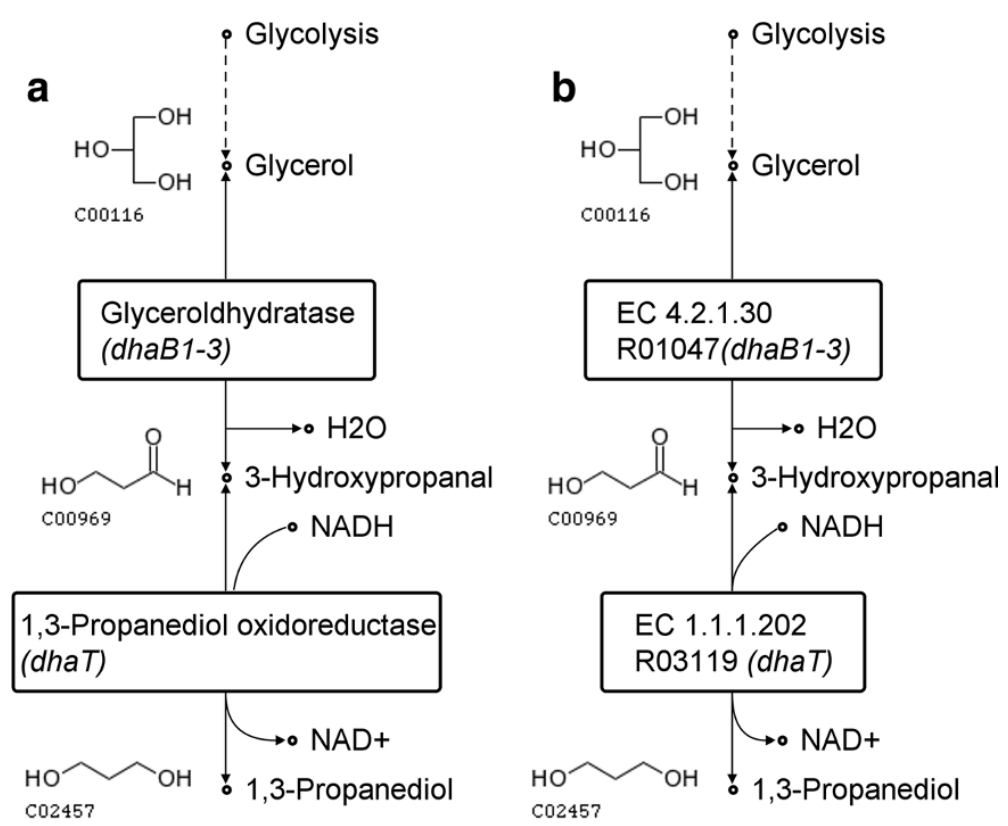

Figure 2 Heterologous pathways for 1,3-propanediol production: (a) the production pathway described in earlier studies, in Escherichia coli [52,53]; (b) the pathway identified by our algorithm in either E. coli or Saccharomyces cerevisiae as the host.

that is, the solution was not unique. In such cases, following maximization of biomass production, the production flux of the target metabolites was further maximized with fixing the maximized biomass production, to obtain a unique flux profile that would generate the target. In the simulations, we adopted a microaerobic condition to screen the target metabolites produced under the biomass maximization condition, in which significantly more metabolites were obtained than

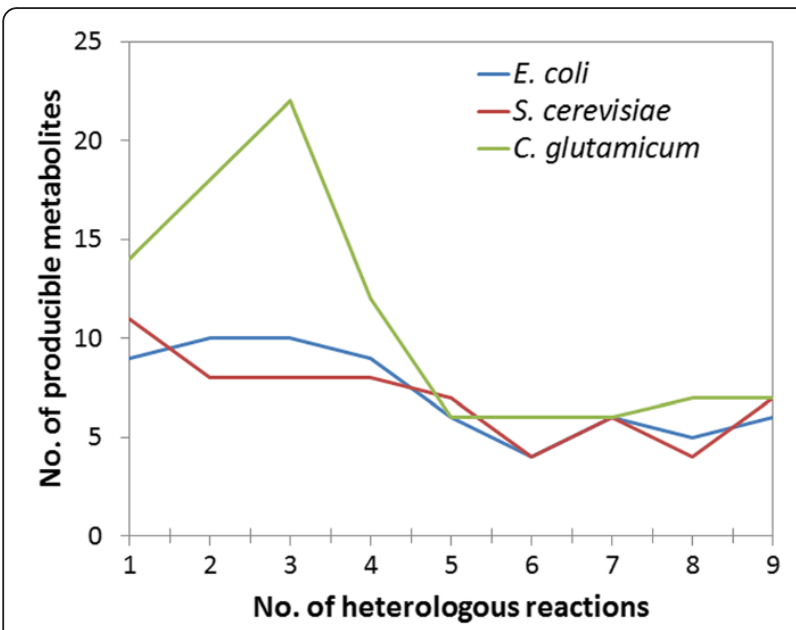

Figure 3 The number of metabolites producible under biomass maximization conditions with the addition of $<10$ heterologous reactions. under anaerobic conditions, and in which all anaerobically produced metabolites were included.

Table 2 lists the representative target metabolites produced under biomass maximization, together with their corresponding heterologous reactions. The mechanisms involved in these reactions can be classified into two categories. One is based on the production of oxygen as a by-product of the targets. Since the simulations were performed under micro-aerobic conditions, oxygen supply increased the biomass production by activating the electron transfer system and facilitating adenosine triphosphate production. Therefore, if the heterologous reactions used to produce the target are accompanied by oxygen production, the target can be produced under minimum biomass production flux. For example, pentane-2,4-dione was produced by introducing a single heterologous reaction into E. coli and S. cerevisiae, whereas two heterologous reactions were necessary to produce this metabolite in C. glutamicum. Vanillin can be produced under the same mechanism by introducing 4 heterologous reactions into the E. coli and C. glutamicum metabolic networks.

Another mechanism is associated with NADH oxidization. Under micro-aerobic conditions, the cellular growth of microorganisms can be limited by NAD regeneration, which is necessary for glycolysis activity, and which occurs through NADH oxidization. Thus, when the heterologous reactions producing the targets are associated with NADH oxidization, these heterologous reactions are activated when the biomass 
Table 2 Examples of producible nonnative metabolites under conditions of maximized biomass production

\begin{tabular}{|c|c|c|c|c|c|}
\hline Nonnative metabolites & Host network & By-product & No. of reaction(s) & Heterologous reaction(s) & EC number \\
\hline \multirow[t]{3}{*}{ Pentane-2,4-dione } & E. coli, S. cerevisiae & Oxygen & 1 & Pentane-2,4-dione + oxygen $\leftrightarrow$ acetate + methylglyoxal & 1.13.11.50 \\
\hline & \multirow[t]{2}{*}{ C. glutamicum } & \multirow[t]{2}{*}{ Oxygen } & \multirow[t]{2}{*}{2} & Glycerone phosphate $\leftrightarrow$ methylglyoxal + orthophosphate & 4.2.3.3 \\
\hline & & & & Pentane-2,4-dione + oxygen $\leftrightarrow$ acetate + methylglyoxal & 1.13 .11 .50 \\
\hline \multirow{4}{*}{$\begin{array}{l}\text { Vanillin (4-hydroxy-3-methoxy } \\
\text {-benzaldehyde) }\end{array}$} & \multirow{4}{*}{$\begin{array}{l}\text { E. coli, } \\
\text { C. glutamicum }\end{array}$} & \multirow[t]{4}{*}{ Oxygen, NADH } & \multirow[t]{4}{*}{4} & Formaldehyde $+\mathrm{NAD}++\mathrm{H}_{2} \mathrm{O} \leftrightarrow$ formate $+\mathrm{NADH}+\mathrm{H}^{+}$ & 1.2.1.46 \\
\hline & & & & 3-Dehydroshikimate $\leftrightarrow$ 3,4-dihydroxybenzoate + $\mathrm{H} 2 \mathrm{O}$ & 4.2.1.118 \\
\hline & & & & $\begin{array}{l}\text { Vanillate }+ \text { oxygen }+\mathrm{NADH}+\mathrm{H}^{+} \leftrightarrow \text { 3,4-dihydroxybenzoate }+\mathrm{NAD} \\
++\mathrm{H}_{2} \mathrm{O}+\text { formaldehyde }\end{array}$ & 1.14.13.82 \\
\hline & & & & $\begin{array}{l}\text { Vanillate + NAD + + H2O } \leftrightarrow \text { 4-hydroxy-3-methoxy-benzaldehyde } \\
+ \text { oxygen + NADH }+\mathrm{H}^{+}\end{array}$ & 1.2.3.9 \\
\hline \multirow[t]{2}{*}{ (R)-Propane-1,2-diol } & \multirow[t]{2}{*}{ E. coli } & \multirow[t]{2}{*}{$\mathrm{NAD}^{+}$} & \multirow[t]{2}{*}{2} & (R)-Lactaldehyde $+\mathrm{NAD}^{+}+\mathrm{H}_{2} \mathrm{O} \leftrightarrow$ (R)-lactate $+\mathrm{NADH}+\mathrm{H}^{+}$ & 1.2.1.23 \\
\hline & & & & (R)-Propane-1,2-diol $+\mathrm{NAD}^{+} \leftrightarrow(\mathrm{R})$-lactaldehyde $+\mathrm{NADH}+\mathrm{H}^{+}$ & 1.1.1.77 \\
\hline \multirow[t]{3}{*}{ 2-Propyn-1-al } & \multirow[t]{3}{*}{ S. cerevisiae } & \multirow[t]{3}{*}{$\mathrm{NAD}^{+}$} & \multirow[t]{3}{*}{3} & 3-Oxopropanoate $\leftrightarrow$ acetaldehyde $+\mathrm{CO}_{2}$ & 4.1.1.- \\
\hline & & & & 3-Oxopropanoate $\leftrightarrow$ propynoate $+\mathrm{H}_{2} \mathrm{O}$ & 4.2.1.27 \\
\hline & & & & 2-Propyn-1-al $+\mathrm{NAD}^{+}+\mathrm{H}_{2} \mathrm{O} \leftrightarrow$ propynoate $+\mathrm{NADH}+\mathrm{H}^{+}$ & 1.2.1.3 \\
\hline \multirow[t]{6}{*}{ Adipate semialdehyde } & \multirow[t]{6}{*}{ E. coli } & \multirow[t]{6}{*}{ NADP+ } & \multirow[t]{6}{*}{6} & Succinyl-CoA + acetyl-CoA ↔ CoA + 3-oxoadipyl-CoA & 2.3.1.174 \\
\hline & & & & (3 S)-3-Hydroxyadipyl-CoA + NAD ${ }^{+} \leftrightarrow 3$-Oxoadipyl-CoA + NADH + H & 1.1.1.35 \\
\hline & & & & 5-Carboxy-2-pentenoyl-CoA + H2O ↔ (3 S)-3-hydroxyadipyl-CoA & 4.2.1.17 \\
\hline & & & & Adipyl-CoA +FAD $\leftrightarrow$ 5-carboxy-2-pentenoyl-CoA + FADH ${ }_{2}$ & 1.3.99.- \\
\hline & & & & Adipate + CoA + ATP $\leftrightarrow$ Adipyl-CoA + AMP + diphosphate & 6.2.1.- \\
\hline & & & & Adipate semialdehyde $+\mathrm{NADP}++\mathrm{H}_{2} \mathrm{O} \leftrightarrow$ adipate $+\mathrm{NADPH}+\mathrm{H}^{+}$ & 1.2.1.4 \\
\hline
\end{tabular}


production is maximized This phenomenon occurs, for example, in the production of (R)-propane-1,2-diol and 2-propyn-1-al.

We also found that some metabolites are produced only by $E$. coli under conditions of maximum biomass production, such as (R)-propane-1,2-diol and adipate semialdehyde. Unlike S. cerevisiae and C. glutamicum, E. coli possesses NAD transhydrogenase, which can convert NADP and NADH to NADPH and NAD respectively (and vice versa). In E. coli cells, the excess NADH is converted to NADPH which can then enter the target production pathway.

\section{Differences in target production capacity among host microorganisms}

While screening for heterologous pathways to produce the target metabolites discussed earlier, differences in production capacity between the three host microorganisms emerged; for example, a group of metabolites was inducible by the addition of heterologous reactions to one of the hosts, but was not produced by the other hosts. To characterize the differences in target production capacity, we categorized the producible metabolites (shown in the Additional files 5, 6, 7) using the KEGG Orthology database [16]. We then performed a chi-square statistical analysis to identify the categories in which the frequency of producible metabolites is significantly higher than expected. Figure 4 shows the 10 categories that demonstrated significant differences $(P<0.001)$. As shown in the figure, metabolites belonging to 5 categories, namely, "tyrosine metabolism," "dioxin degradation," "benzoate degradation," "chlorocyclohexane and chlorobenzene degradation," and "xylene degradation," tended to be producible by $S$. cerevisiae and C. glutamicum but were scarce in E. coli cells.
Similarly, the metabolites in "flavonoid biosynthesis," "phenylpropanoid biosynthesis," and "nicotinate and nicotinamide metabolism" were preferentially generated by E. coli and C. glutamicum. Metabolites assigned to "porphyrin and chlorophyll metabolism" also tended to be produced in C. glutamicum cells. Likewise, the metabolites assigned to "biosynthesis of 12-, 14-, and 16membered macrolides" were produced preferentially in E. coli cells. Such differences in production capabilities result from the different metabolic pathways by which the hosts produce necessary substrates, and from cellular compartmentalization in the yeast strain (which is absent in the bacterial strains).

In yeast cells, the compartments present barriers to metabolite transport. For instance, mitochondrial/cytoplasmic interfaces prohibit the production of certain target metabolites when sugar is used as a carbon source. Similarly, the production of metabolites in the "flavonoid biosynthesis" category was inhibited in yeast cells because the transportation of 4-coumarate between the mitochondria and the cytosol is not permitted; therefore, the yeast strain could not produce $p$-coumaroyl-CoA (required for making chalconoid, an important ingredient in flavonoid biosynthesis). Our genome-scale metabolic model does not account for transportation capabilities between compartments, which are currently unclear for many metabolites, and which might influence the production capacities of target metabolites in real cell systems.

\section{Conclusions}

In conclusion, we developed a computational platform to investigate the extent to which industrial hosts can synthesize nonnative metabolites. Biosynthetic capabilities are evaluated by pathway design and flux calculations. We

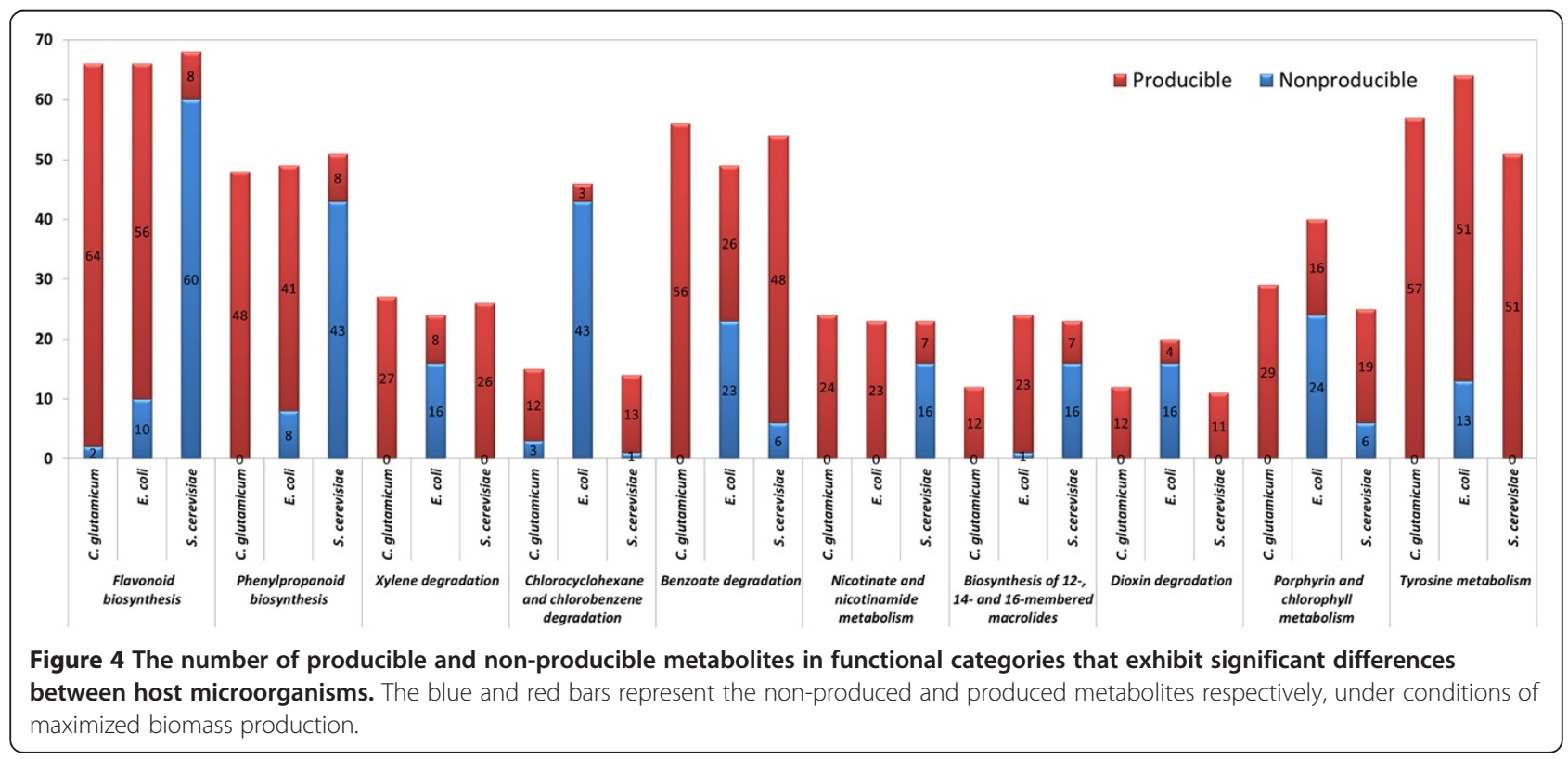


tested our platform using the industrial hosts S. cerevisiae, E. coli, and C. glutamicum as templates. Our results are consistent with those of earlier reports and provide additional alternative heterologous pathways. Producible nonnative metabolites predicted by our platform include industrial chemical compounds such as isoprene, $\alpha$ farnesene, PHB, cadaverine, 1,3-propanediol, 1,2-propanediol, and vanillin. We propose that our platform is applicable to any genome-scale models that simulate cell factories. The platform greatly reduces the time and cost of heterologous pathway searching for target metabolites. Furthermore, appropriate expansions of the proposed system (for example, incorporating reaction irreversibility and source availability of heterologous enzymes), could significantly improve the scope of our system. We believe that this platform will accelerate the rational design of metabolic systems and thereby enhance microbial production of essential metabolites.

\section{Availability and requirements}

The program for our pathway search algorithm is available at

http://www-shimizu.ist.osaka-u.ac.jp/pathway_search. zip. The program is written in Python. After extracting "pathway_search.zip", the tool can be started by double clicking "runningScript.py" or by opening "runningScript.py" in Python IDLE, followed by pressing F5. All connectable nonnative metabolites including heterologous reaction are contained in the iteration folder. The folder input contains the necessary input files for identifying heterologous reactions of nonnative metabolites induced in a specified host.

\section{Additional files}

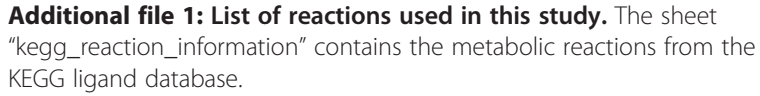

Additional file 2: List of connectable nonnative metabolites when Corynebacterium glutamicum was used as the host. The sheet " $\mathrm{C}$. glutamicum connectable" contains all of the connected metabolites, including heterologous reaction(s), information about gene(s) from the KEGG database and the minimum $K_{m}$ value from the BRENDA database.

Additional file 3: List of connectable nonnative metabolites when Escherichia coli was used as the host. The sheet "E.coli_connectable" contains all of the connected metabolites, including heterologous reaction(s), information about gene(s) from the KEGG database and the minimum $K_{\mathrm{m}}$ value from the BRENDA database.

Additional file 4: List of connectable nonnative metabolites when Saccharomyces cerevisiae was used as the host. The sheet "S. cerevisiae_connectable" contains all of the connected metabolites, including heterologous reaction(s), information about gene(s) from the KEGG database and the minimum $K_{m}$ value from the BRENDA database.

Additional file 5: List of producible nonnative metabolites when Corynebacterium glutamicum was used as the host. The sheet " $\mathrm{C}$. glutamicum_maxTarget" contains all of the producible metabolites under the target maximization condition, including heterologous reaction(s), information about gene(s) from the KEGG database and the minimum $K_{m}$ value from the BRENDA database. The sheet " $\mathrm{C}$.

glutamicum_maxBiomass" contains the producible metabolites under the biomass maximization condition, including heterologous reaction(s), information about gene(s) from the KEGG database and the minimum $K_{m}$ value from the BRENDA database.

Additional file 6: List of producible nonnative metabolites when Escherichia coli was used as the host. The sheet "E.coli_maxTarget" contains all of the producible metabolites under the target maximization condition, including heterologous reaction(s), information about gene(s) from the KEGG database and the minimum $K_{m}$ value from the BRENDA database (nonstandard format). The sheet "E.coli_maxBiomass" contains the producible metabolites under the biomass maximization condition, including heterologous reaction(s), information about gene(s) from the KEGG database and the minimum $K_{m}$ value from the BRENDA database.

Additional file 7: List of producible nonnative metabolite when Saccharomyces cerevisiae was used as the host. The sheet "S. cerevisiae maxTarget" contains all of the producible metabolites under the target maximization condition, including heterologous reaction(s), information about gene(s) from the KEGG database and the minimum $K_{m}$ value from the BRENDA database. The sheet "S.cerevisiae_maxBiomass" contains the producible metabolites under the biomass maximization condition, including heterologous reaction(s), information about gene(s) from the KEGG database and the minimum $K_{m}$ value from the BRENDA database.

Competing interests

No competing interests declared.

\section{Authors' contributions}

SC constructed the algorithm and performed the simulations. CF participated in the design of the study and drafted the manuscript. HS conceived and supervised the study. All authors revised and approved the final manuscript.

\section{Acknowledgment}

This research was partially supported by a Grant-in-Aid for Young Scientists (A) to CF (No. 23680030) from the Japan Society for the Promotion of Science, and JST, ALCA (Advanced Low Carbon Technology Research and Development Program). This work was also supported in part by the Global COE Program of the Ministry of Education, Culture, Sports, Science and Technology of Japan.

\section{Author details}

${ }^{1}$ Department of Biotechnology, Graduate School of Engineering, Osaka University, 2-1 Yamadaoka, Suita, Osaka 565-0871, Japan. ${ }^{2}$ Quantitative Biology Center, RIKEN, 6-2-3 Furuedai, Suita, Osaka 565-0874, Japan. ${ }^{3}$ Department of Bioinformatics Engineering, Graduate School of Information Science and Technology, Osaka University, 1-5 Yamadaoka, Suita, Osaka 565-0871, Japan.

Received: 14 February 2012 Accepted: 24 April 2012

Published: 11 May 2012

\section{References}

1. Dugar D, Stephanopoulos G: Relative potential of biosynthetic pathways for biofuels and bio-based products. Nat Biotechnol 2011, 29:1074-1078.

2. Lee SK, Chou H, Ham TS, Lee TS, Keasling JD: Metabolic engineering of microorganisms for biofuels production: from bugs to synthetic biology to fuels. Curr Opin Biotechnol 2008, 19:556-563.

3. Schneider J, Wendisch VF: Biotechnological production of polyamines by bacteria: recent achievements and future perspectives. Appl Microbiol Biotechnol 2011, 91:17-30.

4. Papini M, Salazar M, Nielsen J: Systems biology of industrial microorganisms. Adv Biochem Eng Biotechnol 2010, 120:51-99.

5. Lee JW, Kim HU, Choi S, Yi J, Lee SY: Microbial production of building block chemicals and polymers. Curr Opin Biotechnol 2011, 22:758-767.

6. McEwen JT, Atsumi S: Alternative biofuel production in non-natural hosts. Curr Opin Biotechnol 2012, 23:1-7.

7. Wang B-wei, Shi A-qin, Tu R, Zhang X-li, Wang Q-H, Bai F-W: BranchedChain Higher Alcohols. Adv Biochem Eng Biotechnol 2012, 128:101-18. 
8. Liu H, Xu Y, Zheng Z, Liu D: 1,3-Propanediol and its copolymers: research, development and industrialization. Biotechnol J 2010, 5:1137-48.

9. Ohya N, Koyama PT: Biopolymers Online. Weinheim, Germany: Wiley-VCH Verlag GmbH \& Co. KGaA; 2005:73-81.

10. Smith KM, Cho K-M, Liao JC: Engineering Corynebacterium glutamicum for isobutanol production. Appl Microbiol Biotechnol 2010, 87:1045-55.

11. Keasling JD: Manufacturing molecules through metabolic engineering. Science (New York, N.Y.) 2010, 330:1355-8.

12. Li H, Zhang G, Deng A, Chen N, Wen T: De novo engineering and metabolic flux analysis of inosine biosynthesis in Bacillus subtilis. Biotechnol Lett 2011, 33:1575-80.

13. Wang C, Yoon S-H, Jang H-J, Chung Y-R, Kim J-Y, Choi E-S, Kim S-W: Metabolic engineering of Escherichia coli for a-farnesene production. Metab Eng 2011, 13:648-655.

14. Gulevich AY, Skorokhodova AY, Sukhozhenko AV, Shakulov RS, Debabov VG: Metabolic engineering of Escherichia coli for 1-butanol biosynthesis through the inverted aerobic fatty acid $\beta$-oxidation pathway. Biotechnol Lett 2011, 34:463-9.

15. Li S, Wen J, Jia X: Engineering Bacillus subtilis for isobutanol production by heterologous Ehrlich pathway construction and the biosynthetic 2-ketoisovalerate precursor pathway overexpression. Appl Microbiol Biotechnol 2011, 91:577-89.

16. Kanehisa M, Araki M, Goto S, Hattori M, Hirakawa M, Itoh M, Katayama T, Kawashima S, Okuda S, Tokimatsu T, Yamanishi Y: KEGG for linking genomes to life and the environment. Nucleic Acids Res 2008, 36:D480-4.

17. Chang A, Scheer M, Grote A, Schomburg I, Schomburg D: BRENDA, AMENDA and FRENDA the enzyme information system: new content and tools in 2009. Nucleic Acids Res 2009, 37:D588-92.

18. Bairoch A: The ENZYME database in 2000. Nucleic Acids Res 2000, 28:304-5.

19. McShan DC, Rao S, Shah I: PathMiner: predicting metabolic pathways by heuristic search. Bioinformatics (Oxford, England) 2003, 19:1692-8

20. Li C, Henry C, Jankowski M, lonita J, Hatzimanikatis V, Broadbelt L: Computational discovery of biochemical routes to specialty chemicals. Chem Eng Sci 2004, 59:5051-5060.

21. Handorf T, Ebenhöh O, Heinrich R: Expanding metabolic networks: scopes of compounds, robustness, and evolution. J Mol Evol 2005, 61:498-512.

22. Rodrigo G, Carrera J, Prather K, Jaramillo A: DESHARKY: automatic design of metabolic pathways for optimal cell growth. Bioinformatics (Oxford, England) 2008, 24:2554-6.

23. Dogrusoz U, Cetintas A, Demir E, Babur O: Algorithms for effective querying of compound graph-based pathway databases. BMC Bioinformatics 2009, 10:376.

24. Henry CS, Broadbelt $\sqcup$, Hatzimanikatis V: Discovery and analysis of novel metabolic pathways for the biosynthesis of industrial chemicals: 3-hydroxypropanoate. Biotechnol Bioeng 2010, 106:462-73.

25. Cho A, Yun H, Park JH, Lee SY, Park S: Prediction of novel synthetic pathways for the production of desired chemicals. BMC Syst Biol 2010, 4:35

26. Varma A, Palsson BO: Path finding methods accounting for stoichiometry in metabolic networks. Genome Biol 2011, 12:R49.

27. Yousofshahi M, Lee K, Hassoun S: Probabilistic pathway construction. Metab Eng 2011, 13:435-44.

28. Flórez LA, Gunka K, Polanía R, Tholen S, Stülke J: SPABBATS: A pathwaydiscovery method based on Boolean satisfiability that facilitates the characterization of suppressor mutants. BMC Syst Biol 2011, 5:5.

29. Moriya Y, Shigemizu D, Hattori M, Tokimatsu T, Kotera M, Goto S, Kanehisa M: PathPred: an enzyme-catalyzed metabolic pathway prediction server Nucleic Acids Res 2010, 38:W138-43.

30. Pharkya P, Burgard AP, Maranas CD: OptStrain: A computational framework for redesign of microbial production systems. Genome Res 2004, 14:2367-2376.

31. Burgard AP, Pharkya P, Maranas CD: Optknock: a bilevel programming framework for identifying gene knockout strategies for microbial strain optimization. Biotechnol Bioeng 2003, 84:647-57.

32. Shen CR, Lan El, Dekishima Y, Baez A, Cho KM, Liao JC: Driving forces enable high-titer anaerobic 1-butanol synthesis in Escherichia coli. Appl Environ Microbiol 2011, 77:2905-15.

33. Clomburg JM, Gonzalez R: Metabolic engineering of Escherichia coli for the production of 1,2-propanediol from glycerol. Biotechnol Bioeng 2011, 108:867-79.

34. Juminaga D, Baidoo EEK, Redding-Johanson AM, Batth TS, Burd H, Mukhopadhyay A, Petzold CJ, Keasling JD: Modular engineering of L- tyrosine production in Escherichia coli. Appl Environ Microbiol 2012, 78:89-98.

35. Becker J, Wittmann C: Bio-based production of chemicals, materials and fuels -Corynebacterium glutamicum as versatile cell factory. Curr Opin Biotechnol 2011, 23:1-10

36. Hong K-K, Nielsen J: Metabolic engineering of Saccharomyces cerevisiae: a key cell factory platform for future biorefineries. Cell Mol Life Sci 2012, 69:1-20. CMLS.

37. Christina SD: The Metabolic Pathway Engineering Handbook: Fundamentals. 1st edition. USA: CRC Press, Taylor\& Francis Group, LLC; 2010. Section V.

38. Zhang Y, Zhu Y, Zhu Y, Li Y: The importance of engineering physiological functionality into microbes. Trends Biotechnol 2009, 27:664-72.

39. Mo ML, Palsson BO, Herrgård MJ: Connecting extracellular metabolomic measurements to intracellular flux states in yeast. BMC Syst Biol 2009, 3:37

40. Reed JL, Vo TD, Schilling CH, Palsson BO: An expanded genome-scale model of Escherichia coli K-12 (iJR904 GSM/GPR). Genome Biol 2003, 4:R54.

41. Shinfuku Y, Sorpitiporn N, Sono M, Furusawa C, Hirasawa T, Shimizu H: Development and experimental verification of a genome-scale metabolic model for Corynebacterium glutamicum. Microb Cell Fact 2009, 8:43.

42. Enzyme Nomenclature., [http://www.chem.qmul.ac.uk/iubmb/enzyme/]

43. Orth JD, Thiele I, Palsson BØ: What is flux balance analysis? Nat Biotechnol 2010, 28:245-8.

44. Kauffman KJ, Prakash P, Edwards JS: Advances in flux balance analysis. Curr Opin Biotechnol 2003, 14:491-6.

45. Schuetz R, Kuepfer L, Sauer U: Systematic evaluation of objective functions for predicting intracellular fluxes in Escherichia coli. Mol Syst Biol 2007, 3:119.

46. GLPK: GNU Linear Programming Kit. [http://www.gnu.org/software/glpk/].

47. Zhao Y, Yang J, Qin B, Li Y, Sun Y, Su S, Xian M: Biosynthesis of isoprene in Escherichia coli via methylerythritol phosphate (MEP) pathway. Appl Microbiol Biotechnol 2011, 90:1915-22

48. Mahishi LH, Tripathi G, Rawal SK: Poly(3-hydroxybutyrate) (PHB) synthesis by recombinant Escherichia coli harbouring Streptomyces aureofaciens PHB biosynthesis genes: effect of various carbon and nitrogen sources. Microbiol Res 2003, 158:19-27.

49. Kind S, Jeong WK, Schröder H, Wittmann C: Systems-wide metabolic pathway engineering in Corynebacterium glutamicum for bio-based production of diaminopentane. Metab Eng 2010, 12:341-51.

50. Lindahl A-L, Olsson ME, Mercke P, Tollbom O, Schelin J, Brodelius M, Brodelius PE: Production of the artemisinin precursor amorpha-4,11-diene by engineered Saccharomyces cerevisiae. Biotechnol Lett 2006, 28:571-80.

51. Wallaart TE, Bouwmeester HJ, Hille J, Poppinga L, Maijers NC: Amorpha4,11-diene synthase: cloning and functional expression of a key enzyme in the biosynthetic pathway of the novel antimalarial drug artemisinin. Planta 2001, 212:460-5.

52. Nakamura CE, Whited GM: Metabolic engineering for the microbial production of 1,3-propanediol. Curr Opin Biotechnol 2003, 14:454-9.

53. Cameron DC, Altaras NE, Hoffman ML, Shaw AJ: Metabolic engineering of propanediol pathways. Biotechnol Prog 1998, 14:116-25.

54. Inui M, Kawaguchi H, Murakami S, Vertès AA, Yukawa H: Metabolic engineering of Corynebacterium glutamicum for fuel ethanol production under oxygen-deprivation conditions. J Mol Microbiol Biotechnol 2004, 8:243-54.

55. Nielsen DR, Yoon S-H, Yuan CJ, Prather KLJ: Metabolic engineering of acetoin and meso-2, 3-butanediol biosynthesis in E. coli. Biotechnol J 2010, 5:274-84

56. Altaras NE, Cameron DC: Metabolic engineering of a 1,2-propanediol pathway in Escherichia coli. Appl Environ Microbiol 1999, 65:1180-5.

57. Lee W, Dasilva NA: Application of sequential integration for metabolic engineering of 1,2-propanediol production in yeast. Metab Eng 2006, 8:58-65.

58. Niu W, Draths KM, Frost JW: Benzene-free synthesis of adipic acid. Biotechnol Prog 2002, 18:201-11.

59. Edwards JS, Palsson BO: The Escherichia coli MG1655 in silico metabolic genotype: its definition, characteristics, and capabilities. Proc Natl Acad Sci U S A 2000, 97:5528-33.

60. Varma A, Palsson BO: Stoichiometric flux balance models quantitatively predict growth and metabolic by-product secretion in wild-type Escherichia coli W3110. Appl Environ Microbiol 1994, 60:3724-31. 
61. Feist AM, Palsson BO: The biomass objective function. Curr Opin Microbiol 2010, 13:344-9.

62. Edwards JS, Ibarra RU, Palsson BO: In silico predictions of Escherichia coli metabolic capabilities are consistent with experimental data. Nat Biotechnol 2001, 19:125-30.

63. Fong SS, Marciniak JY, Palsson BØ: Description and interpretation of adaptive evolution of Escherichia coli K-12 MG1655 by using a genome-scale in silico metabolic model. J Bacteriol 2003, 185:6400-8.

64. Edwards JS, Palsson BO: Metabolic flux balance analysis and the in silico analysis of Escherichia coli K-12 gene deletions. BMC Bioinformatics 2000 $1: 1$

65. Soyer OS, Pfeiffer T: Evolution under fluctuating environments explains observed robustness in metabolic networks. PLoS Comput Biol 2010, 6.

66. Cornelius SP, Lee JS, Motter AE: Dispensability of Escherichia coli's latent pathways. Proc Natl Acad Sci U S A 2011, 108:3124-9.

67. Gerdes SY, Scholle MD, Campbell JW, Balázsi G, Ravasz E, Daugherty MD, Somera AL, Kyrpides NC, Anderson I, Gelfand MS, Bhattacharya A, Kapatral V, D'Souza M, Baev MV, Grechkin Y, Mseeh F, Fonstein MY, Overbeek R, Barabási A-L, Oltvai ZN, Osterman AL: Experimental determination and system level analysis of essential genes in Escherichia coli MG1655. J Bacteriol 2003, 185:5673-84

doi:10.1186/1471-2105-13-93

Cite this article as: Chatsurachai et al: An in silico platform for the design of heterologous pathways in nonnative metabolite production. BMC Bioinformatics 2012 13:93.

\section{Submit your next manuscript to BioMed Central and take full advantage of:}

- Convenient online submission

- Thorough peer review

- No space constraints or color figure charges

- Immediate publication on acceptance

- Inclusion in PubMed, CAS, Scopus and Google Scholar

- Research which is freely available for redistribution 